

\title{
Multiliteracies in the Course of Turkish as a Foreign Language in Tertiary Education: Didactic Practices
}

Nikolaos Liazos \& Dimitra Markati

To Link this Article: http://dx.doi.org/10.46886/MAJESS/v7-i1/6445

DOI: 10.46886/MAJESS/v7-i1/6445

Received: 21 April 2019, Revised: 25 May 2019, Accepted: 10 June 2019

Published Online: 29 June 2019

In-Text Citation: (Liazos \& Markati, 2019)

To Cite this Article: Liazos N., \& Markati D. (2019). Multiliteracies in the course of Turkish as a foreign language in Tertiary Education: didactic practices. Multilingual Academic Journal of Education and Social Sciences, 7(1), 97-109.

\section{Copyright: (C) The Authors 2019}

Published by Knowledge Words Publications (www.kwpublications.com)

This article is published under the Creative Commons Attribution (CC BY 4.0) license. Anyone may reproduce, distribute, translate and create derivative works of this article (for both commercial and non-commercial purposes), subject to full attribution to the original publication and authors. The full terms of this license may be seen at: http://creativecommons.org/licences/by/4.0/legalcode

Vol. 7, No. 1, 2019, Pg. 97 - 109

https://kwpublications.com/journals/journaldetail/MAJESS

JOURNAL HOMEPAGE

Full Terms \& Conditions of access and use can be found at https://kwpublications.com/pages/detail/publication-ethics 








 Практіке́ৎ
}









\begin{abstract}
Taking into consideration the outcomes of previews papers they describe the modern polymorphic environments and highlight the particular needs of greek-speaking students the present paper focuses on the presentation of a specific teaching model of literature texts in the course of turkish language with benchmark the modern theory's principals of multiliteracy. In this context promoted activities focusing on self-guided learning and the use of multimedia tools. For the needs of this research was chosen to present and compare two different methodological approaches teaching the same literary text implemented in the turkish language course at the department of balkan, slavic and oriental studies of the university of macedonia. On a second level, it was considered important to evaluate the teaching process described by students as essential co-makers.
\end{abstract}

Keywords: Turkish as a Foreign Language, Multiliteracies, Tertiary Education, Multimedia, Didactic Practices.


















































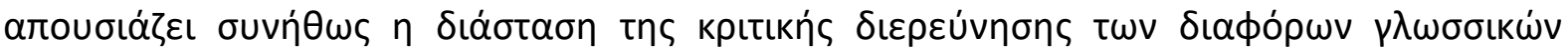



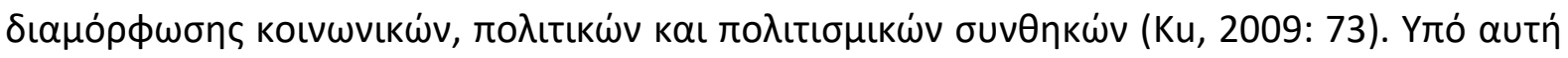









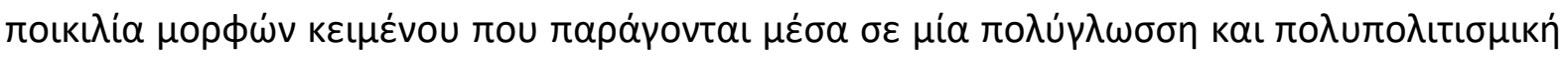

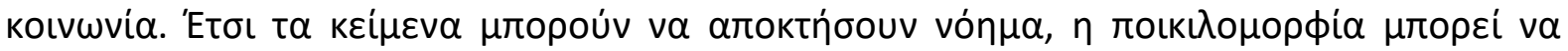






























$\lambda$ ívou.



















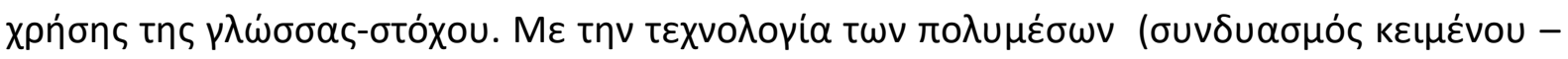














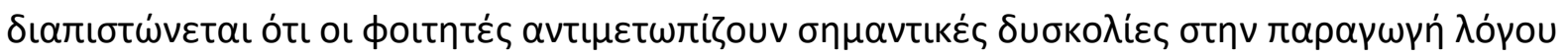









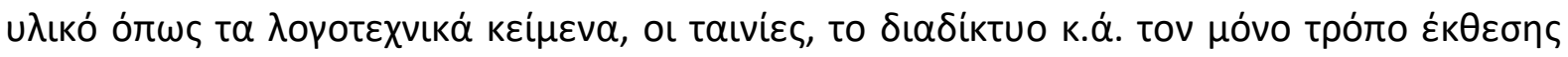

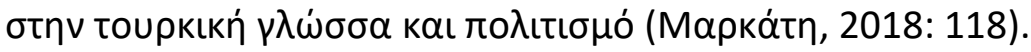



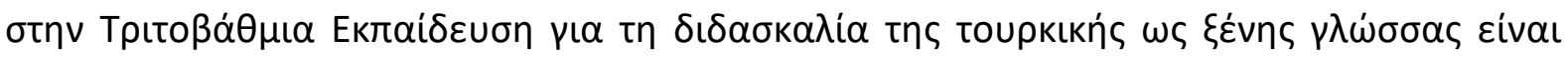
$\alpha v \theta \circ \lambda$ $\pi \alpha \rho o v \sigma i \alpha \sigma \eta \eta$











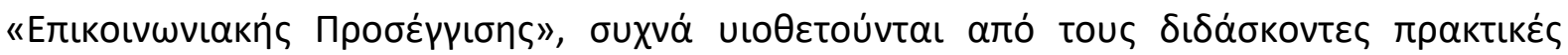

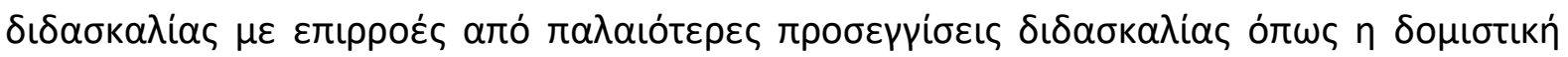

























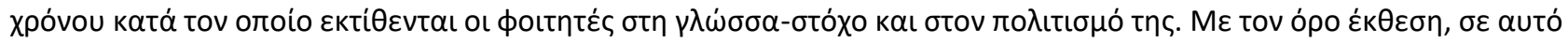





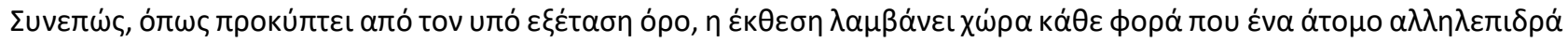




акроати́s.








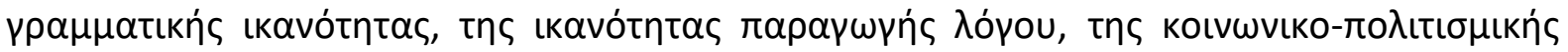

























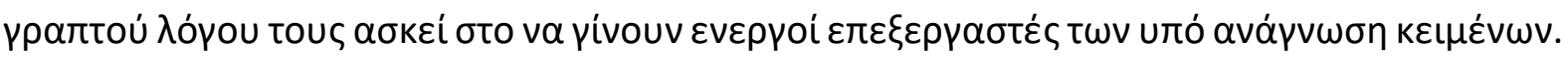













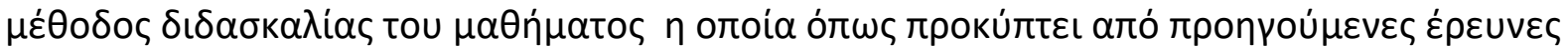















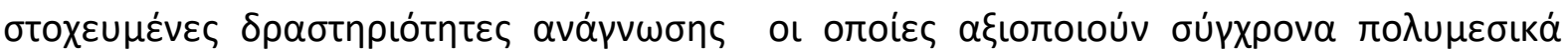







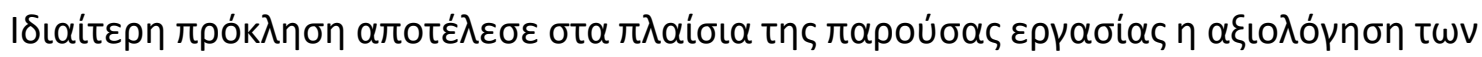

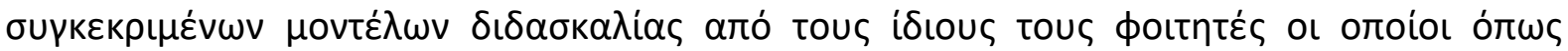



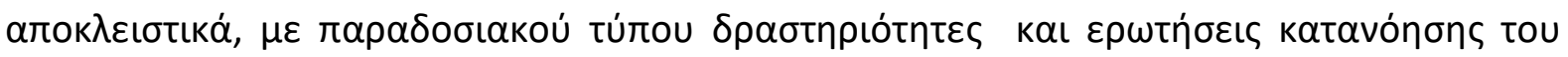




$\varepsilon \mu \pi \varepsilon\llcorner\rho i ́ \alpha$ touৎ.

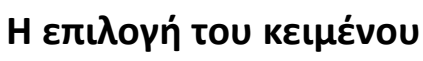















ávepyol.

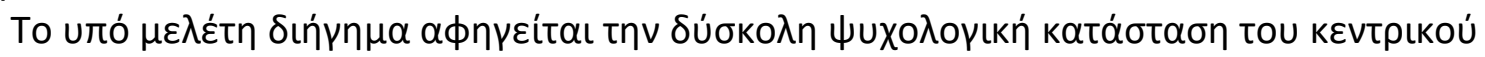



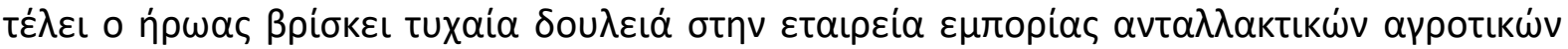

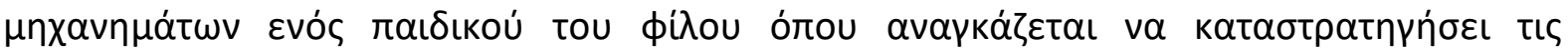

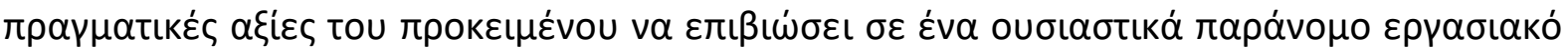





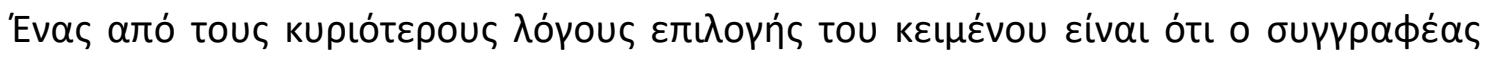





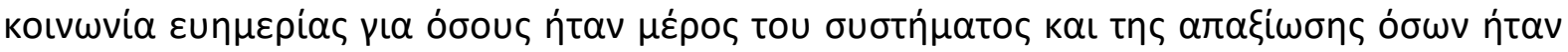







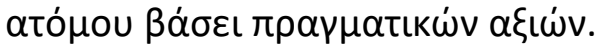



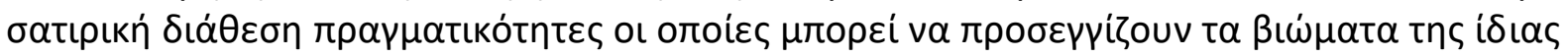



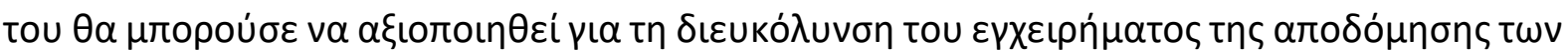





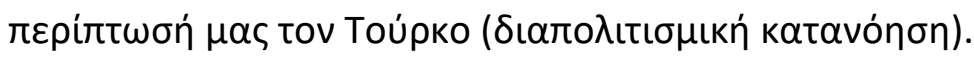

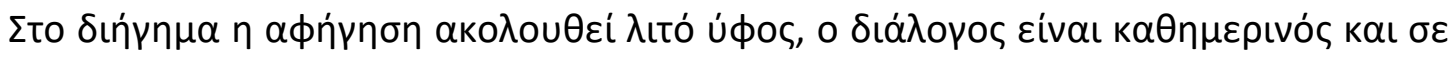





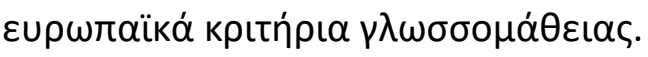





























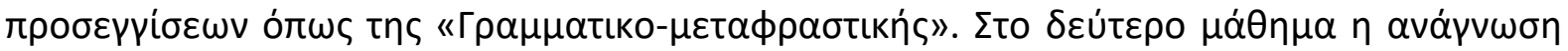




















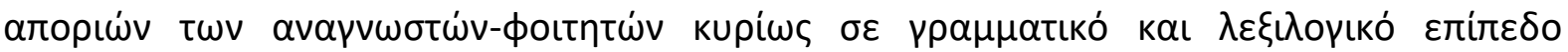



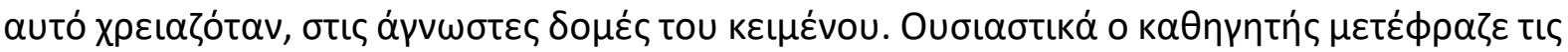

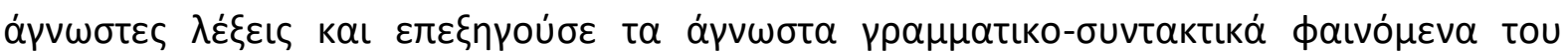

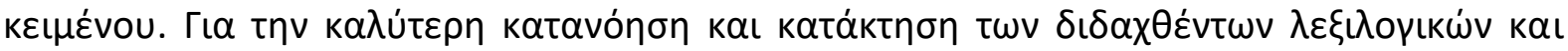



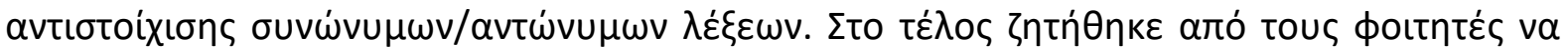



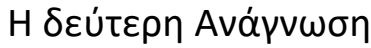



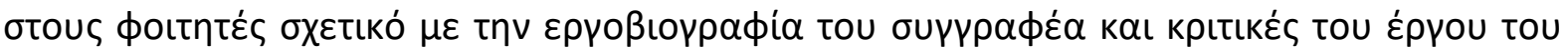





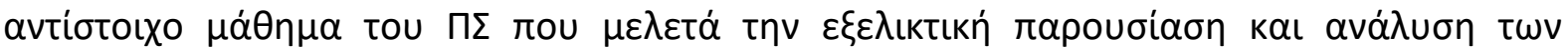







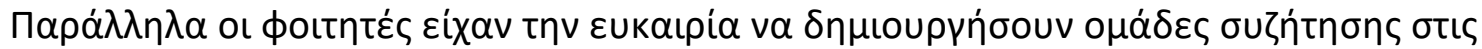






2014: 546).











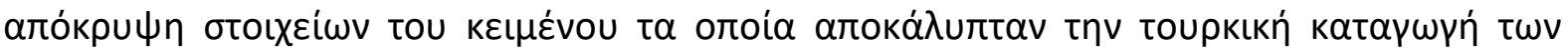



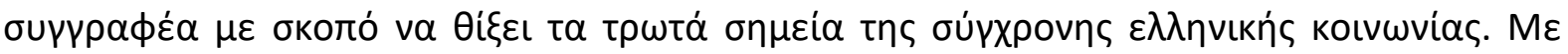













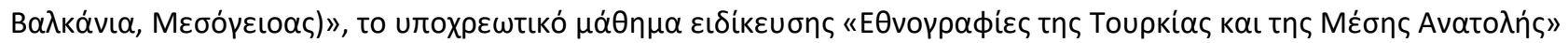








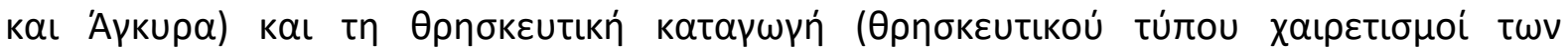

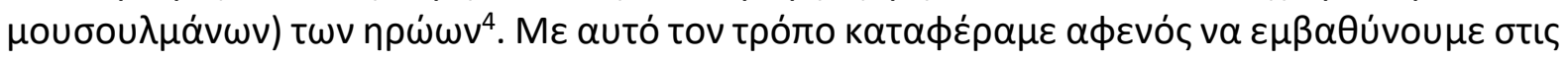

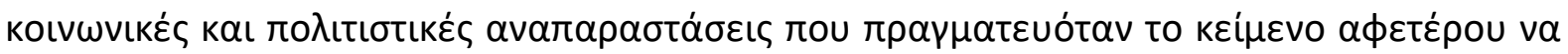

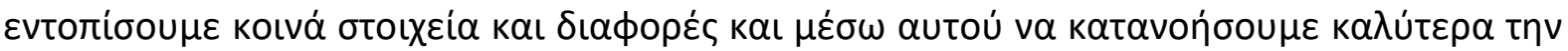

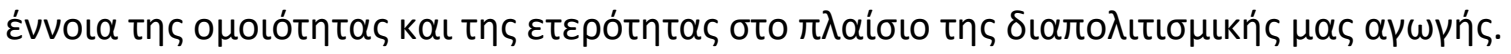

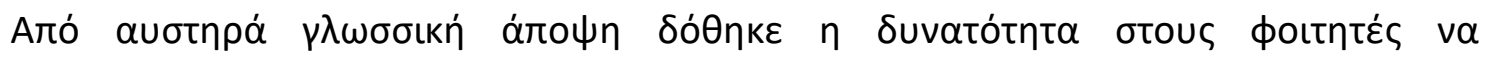

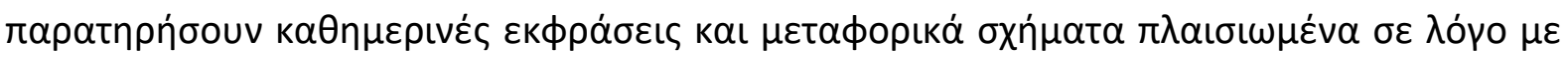

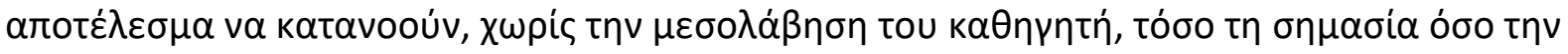

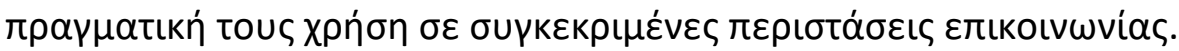

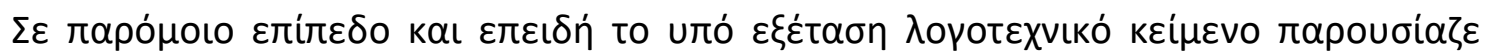

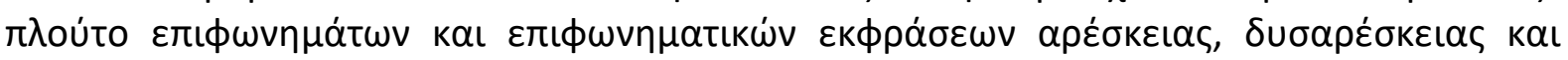

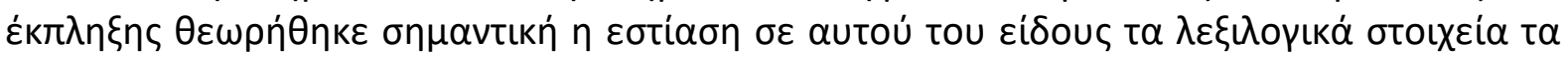

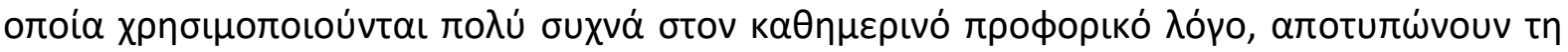

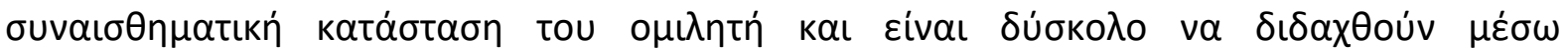
отоเоU



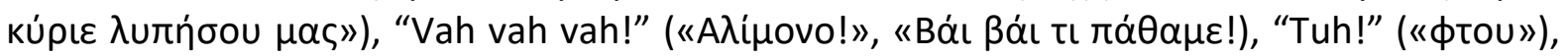

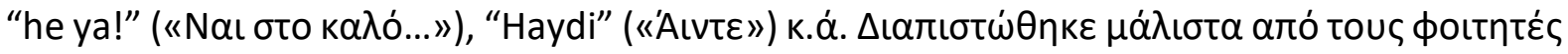



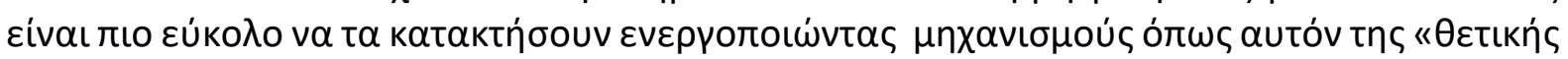
$\pi \alpha \rho \varepsilon \mu \beta о \lambda \eta \dot{\varsigma » ~(М \pi \varepsilon ́ \lambda \lambda \alpha, ~ 2007: ~ 32) . ~}$





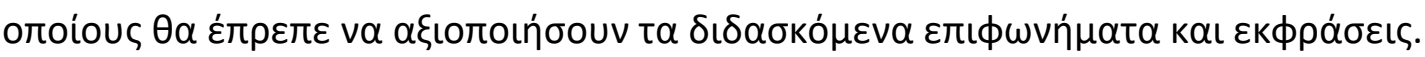



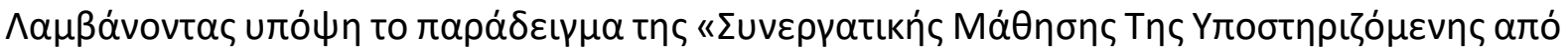
Үло入оүьбтn'" (Computer Supported Collaborative Learning) (Stahl, Koschmann \& Suthers,





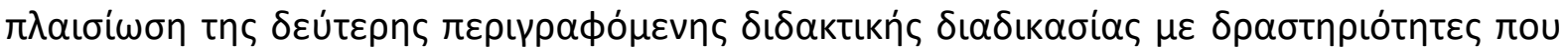









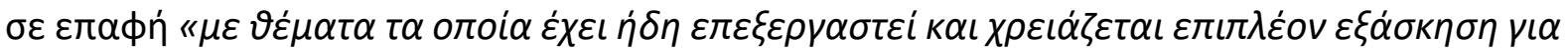

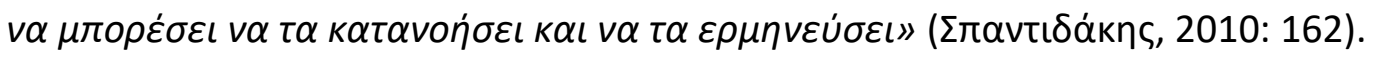

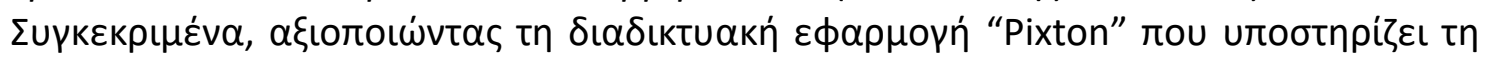

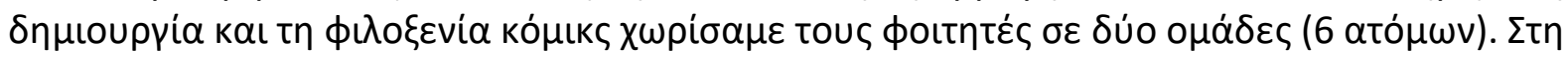

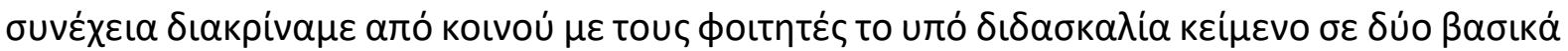

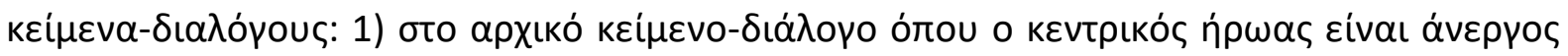

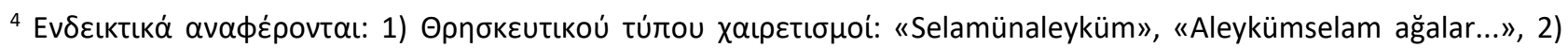

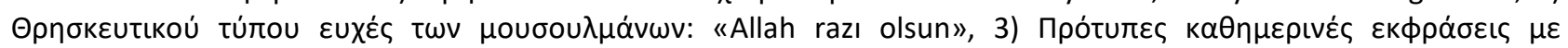

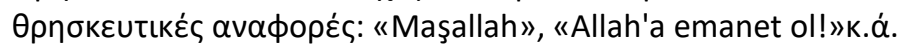




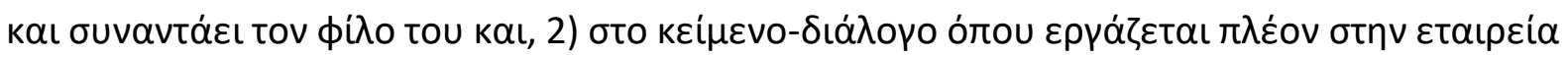

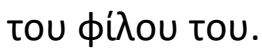

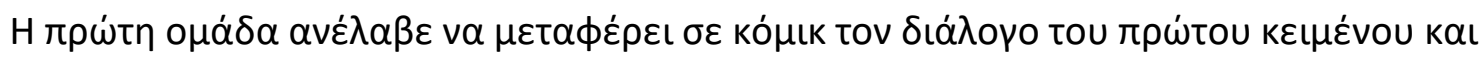

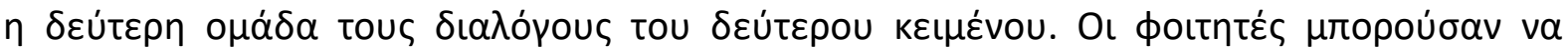

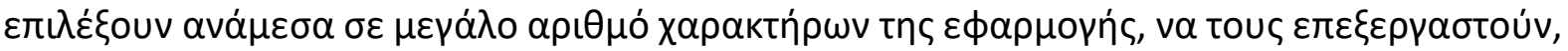

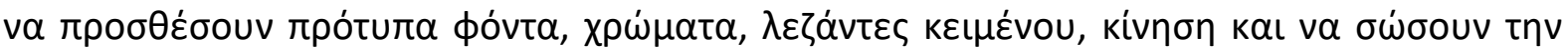

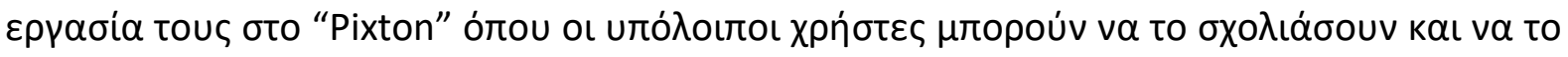

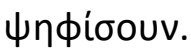

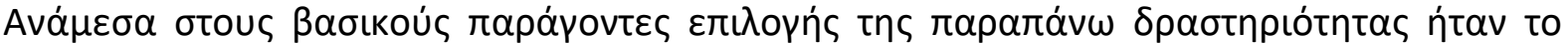

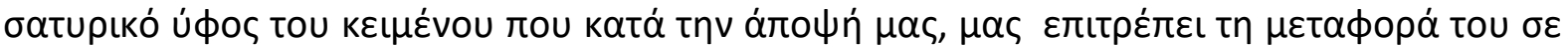

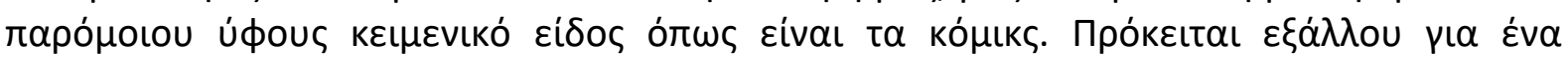

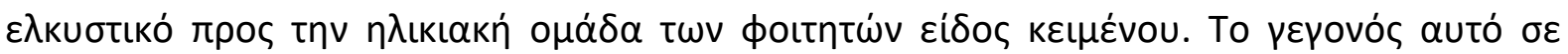

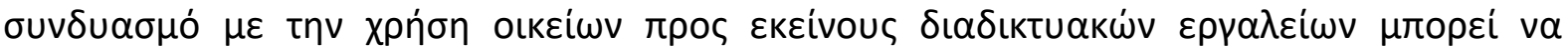

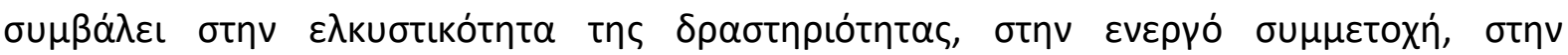

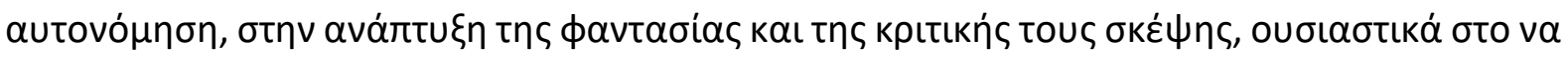

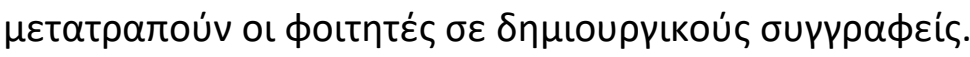

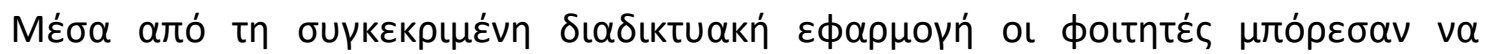

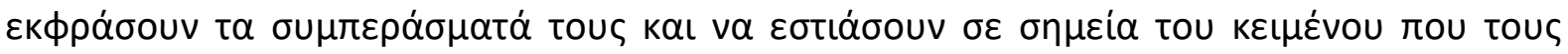

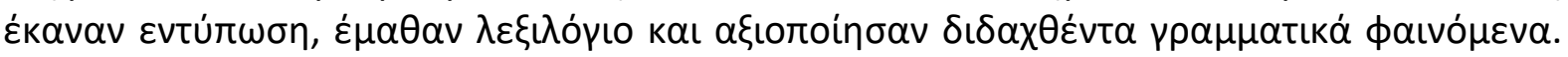

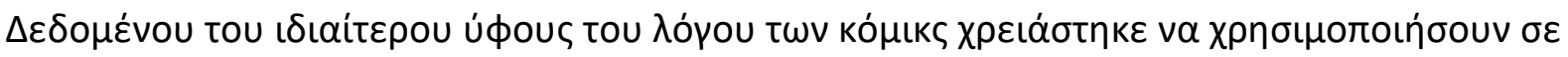

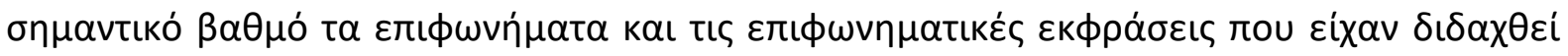
$\pi \rho o \eta \gamma o u \mu \varepsilon ́ v \omega \varsigma$.

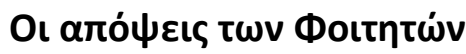

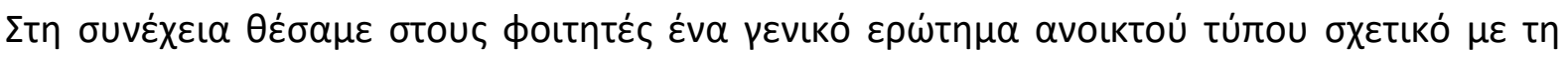

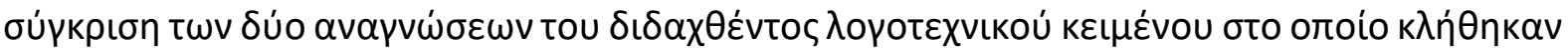

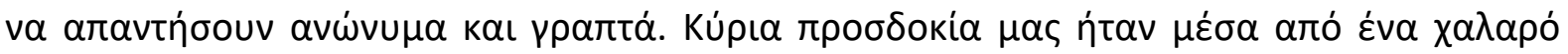

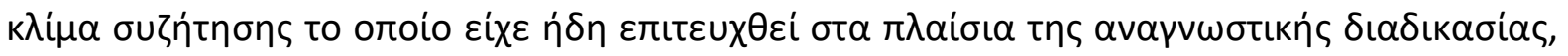

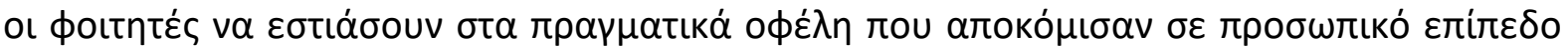

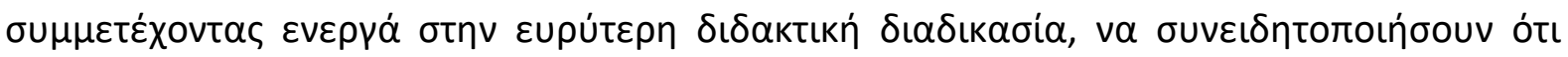

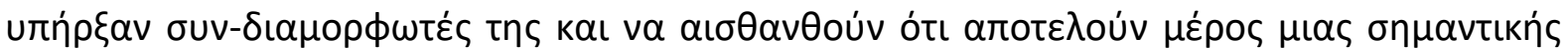

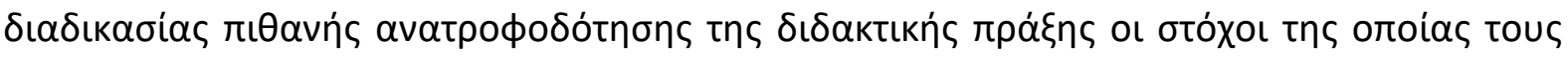

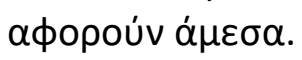

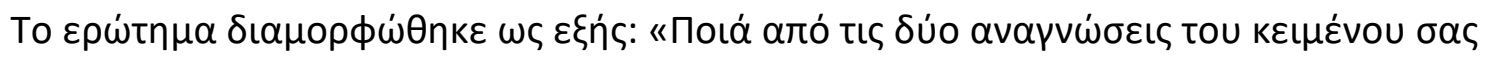

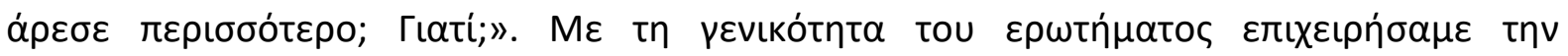

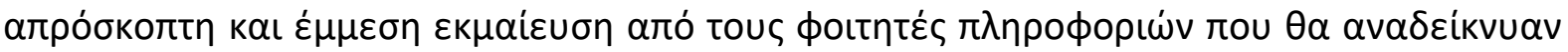

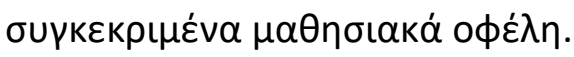

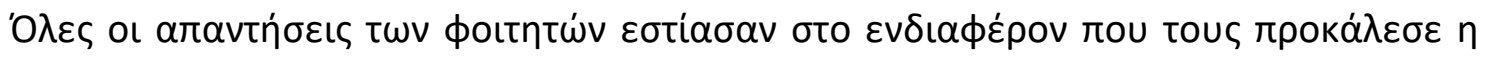

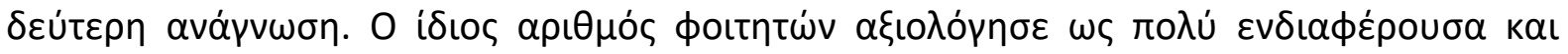

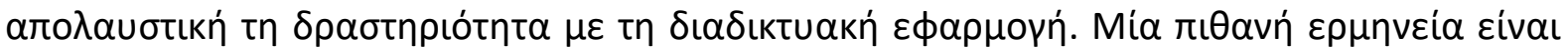

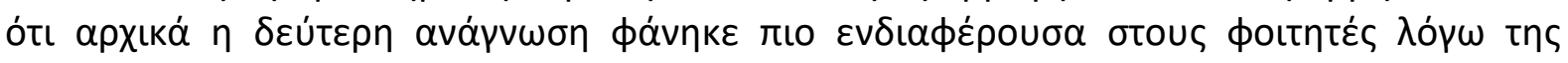

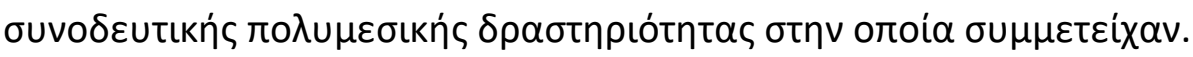

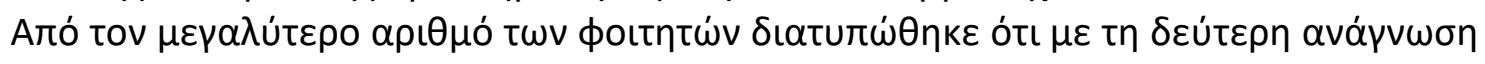

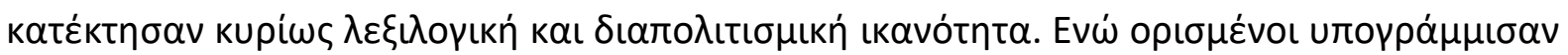

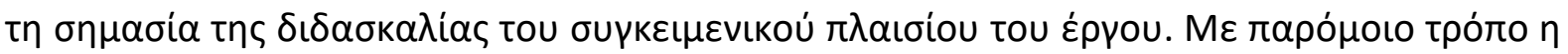

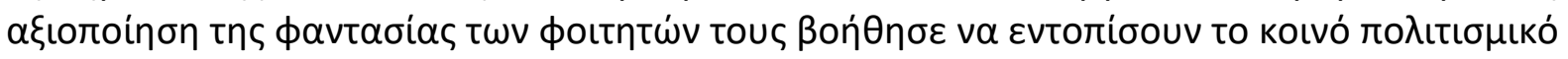




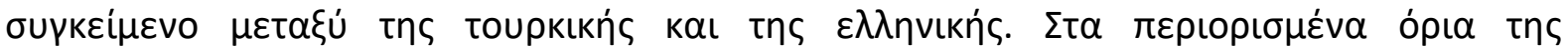

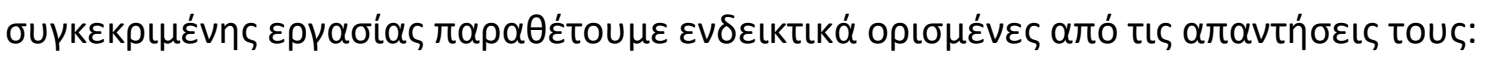

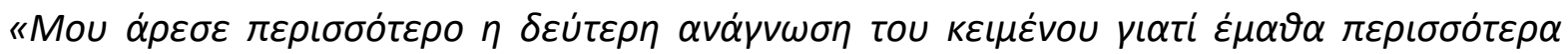

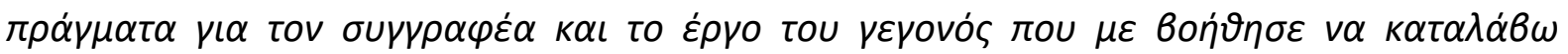

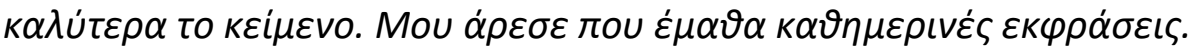

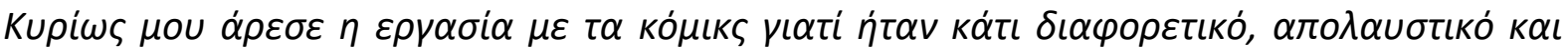

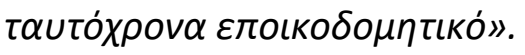

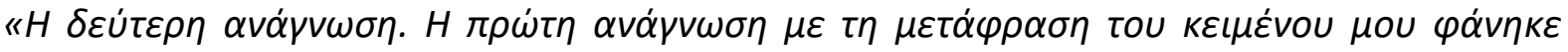

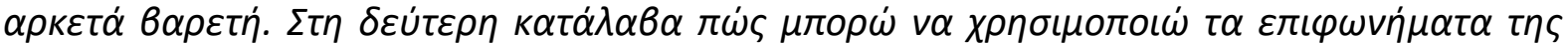

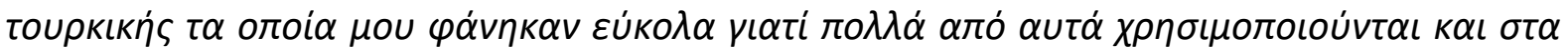

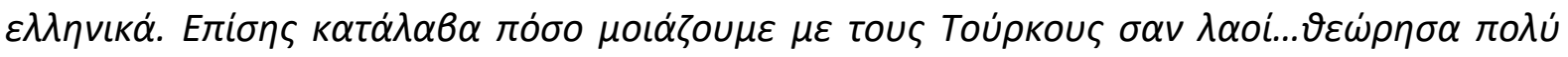

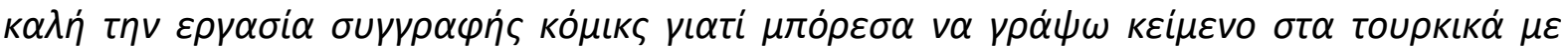

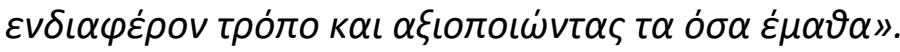

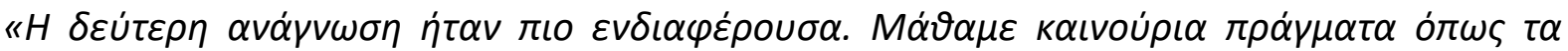

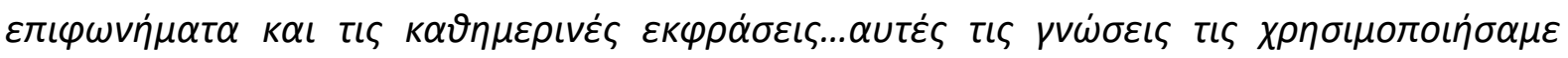

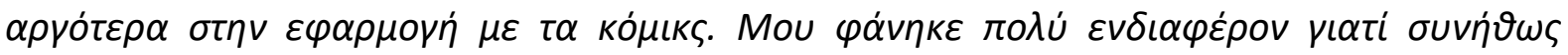

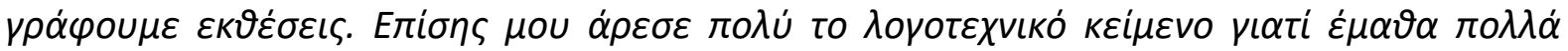

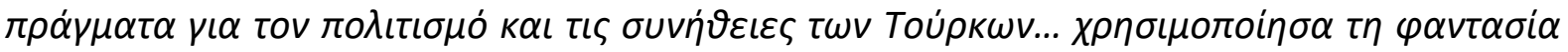
mou».

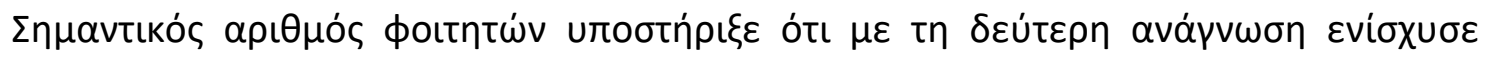

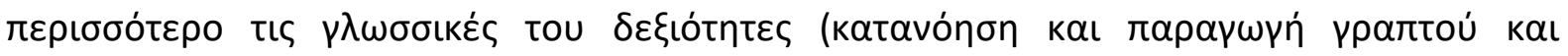

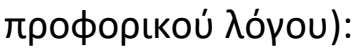

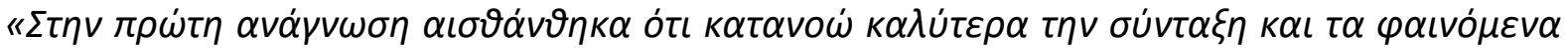

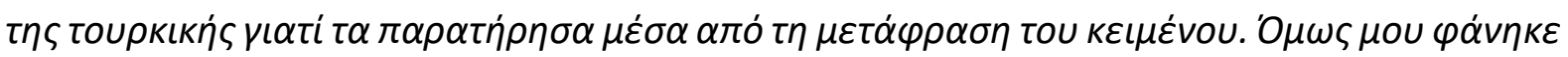

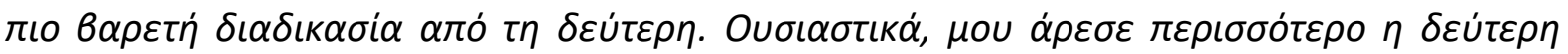

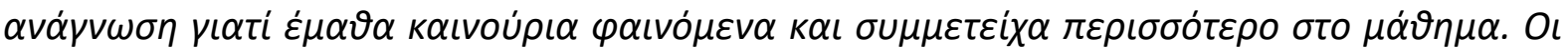

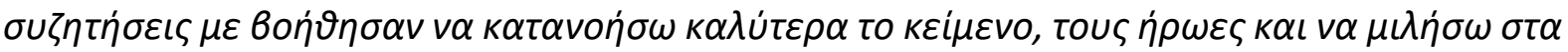

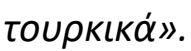

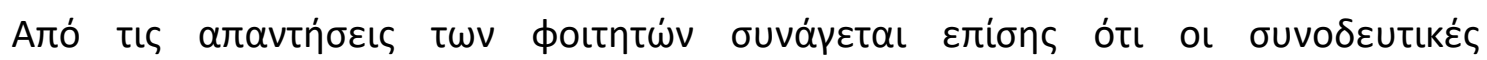

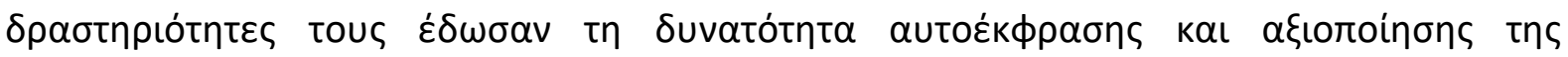

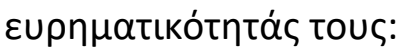

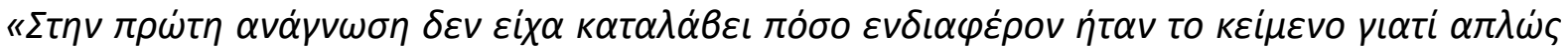

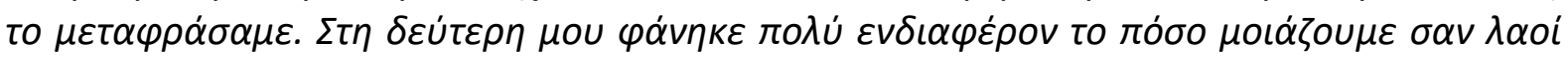

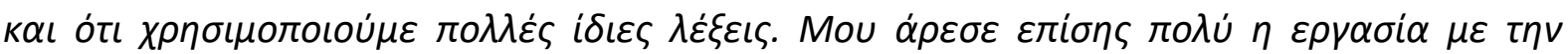

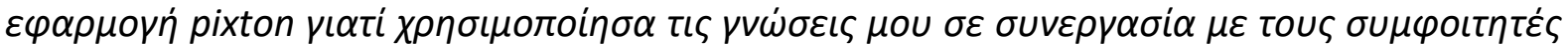

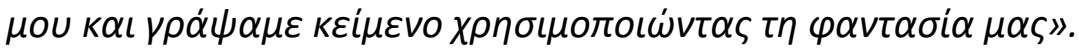

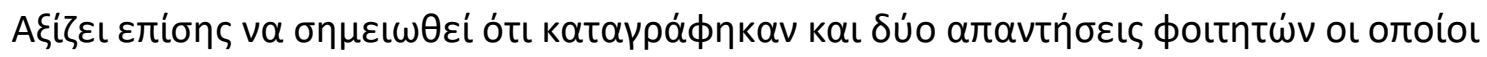

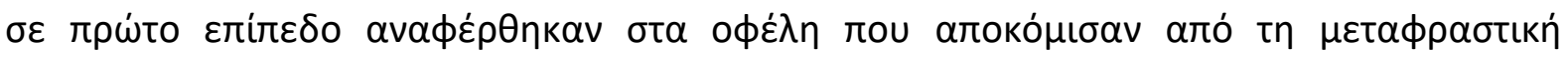

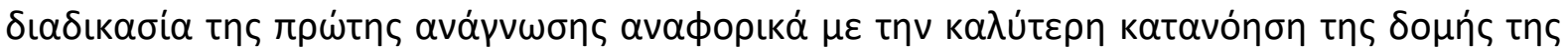

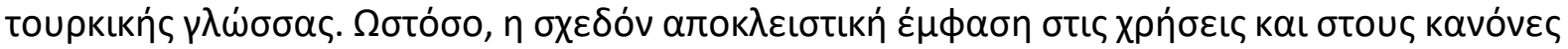

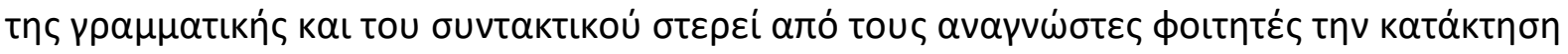

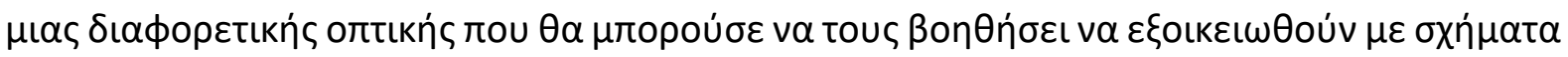

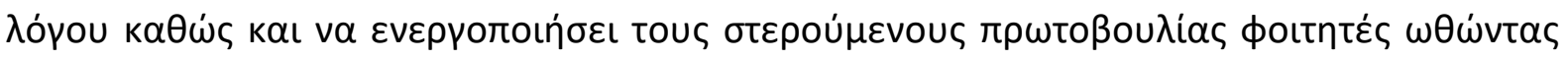

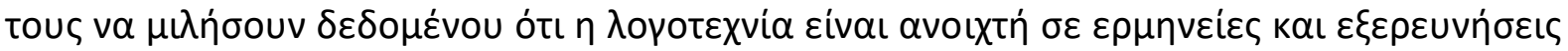

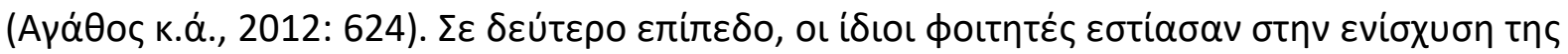




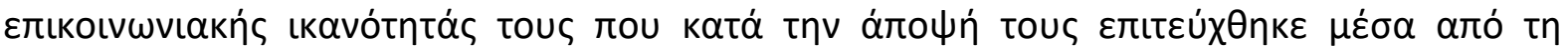

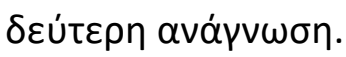

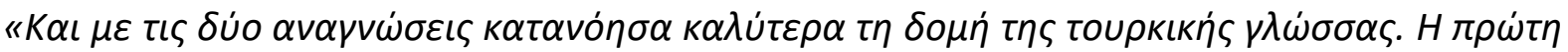

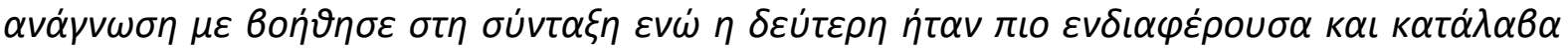

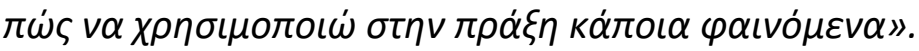

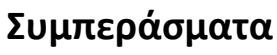

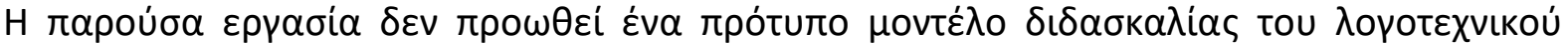

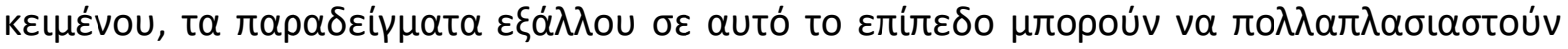

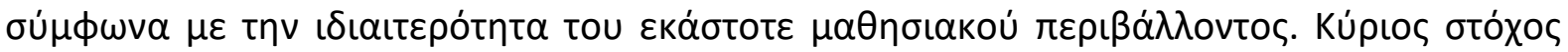

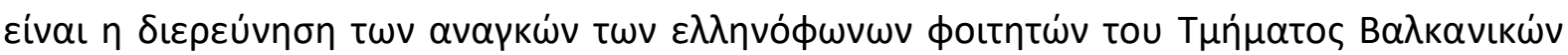

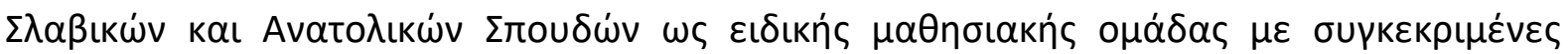

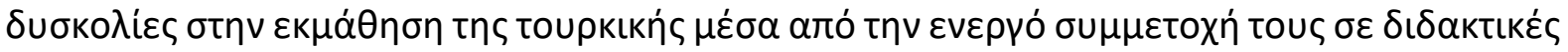

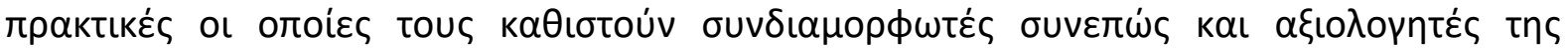

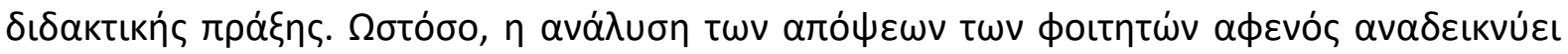

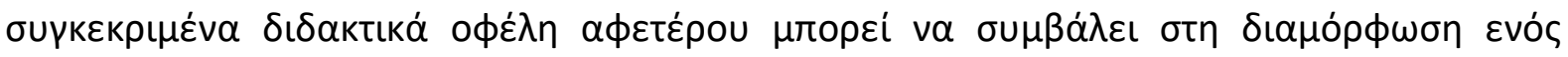

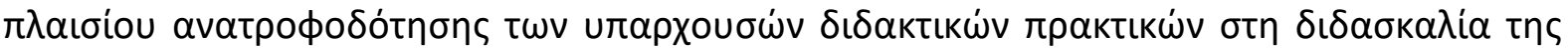

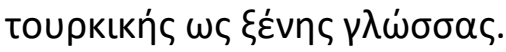

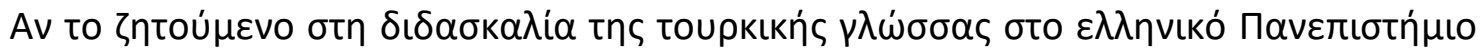

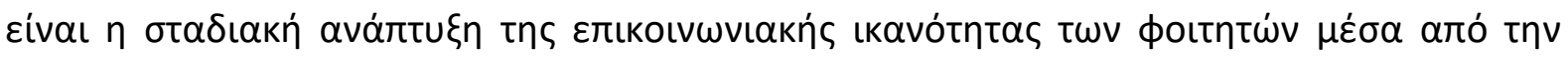

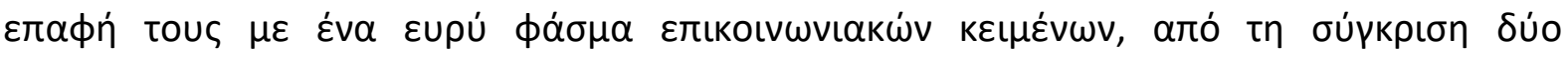

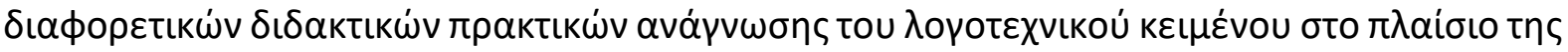

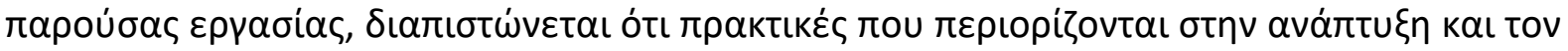

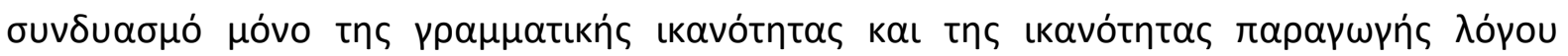

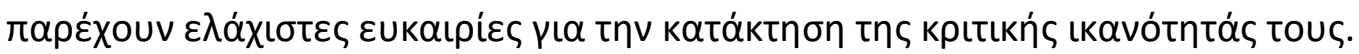

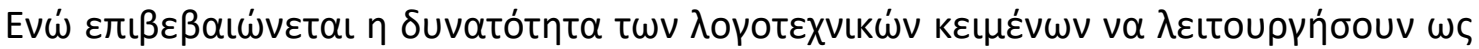

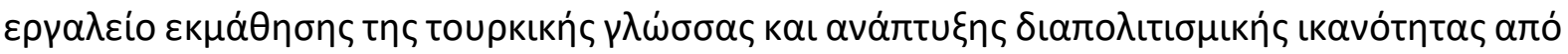

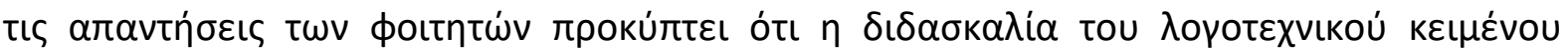

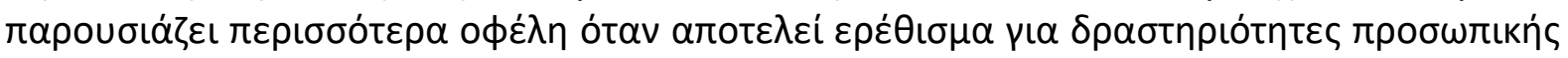

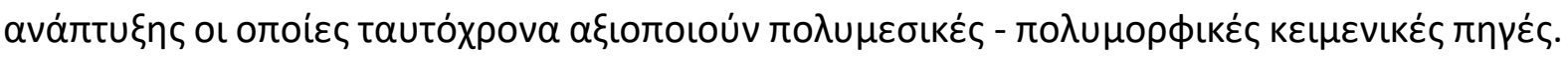

\section{References}

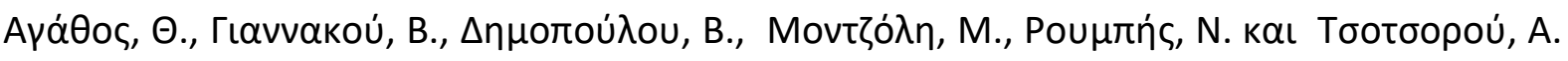

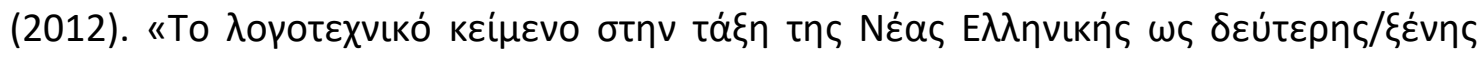

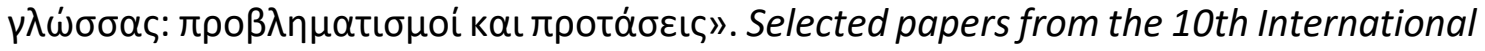
Conference of Greek Linguistics, Gavriilidou, Z., Efthymiou, A., Thomadaki, E., \& Kambakis-Vougiouklis, P. (eds.), Democritus University of Thrace, Komotini, 623-630.

Albers, P., \& Harste, J. C. (2007). The arts, new literacies and multimodality. English Education, $40(1), 6-20$.

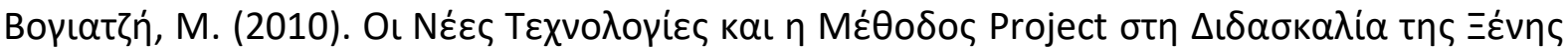

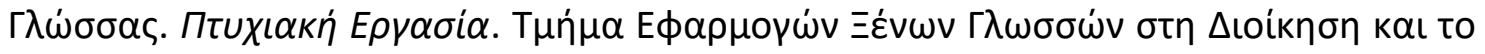

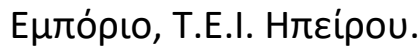

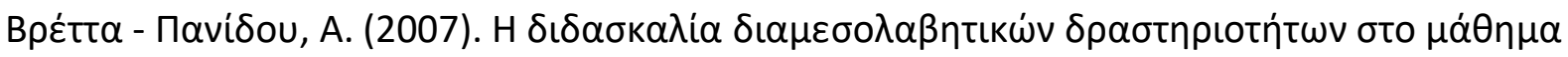

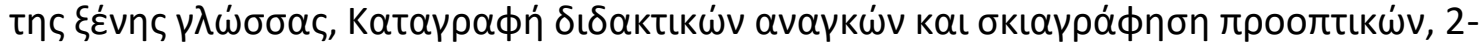

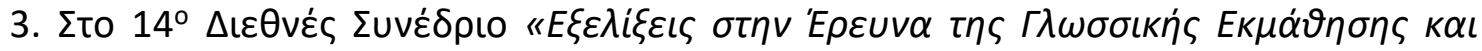




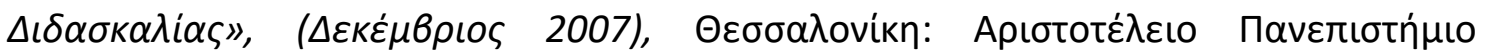

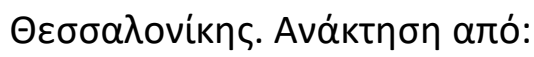

www.enl.auth.gr/gala/ppts/vretta.ppt.

Bryan, J. (2000). Methods of text and discourse analysis, London: Sage, 26-35.

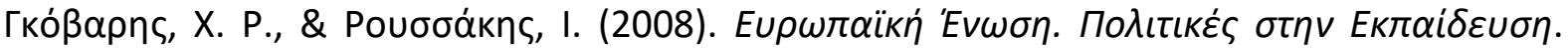

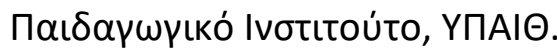

Darancik, Y. (2012). Yabanci Dili Gelistirme Yontemi' ile Yabanci Dil Derslerinde Okuma Becerisinin Gelistirilmesi. C.U. Sosyal Bilimler Enstitusu Dergisi, 21(2), 255-268.

Demircioglu, S. (2010). Teaching English vocabulary to young learners via drama. Procedia Social and Behavioral Sciences, 2, 439-443. Available online at www. Science direct. com.

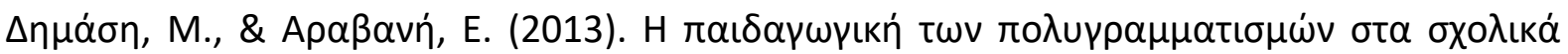

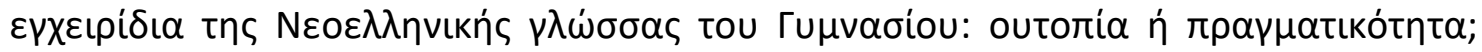
MAJESS, 2, 55-64.

Gocer, A. (2015). Turkcenin Yabanci Dil Olarak Ogretiminde Konusma Becerisinin Kazandirilmasi. Trakya Universitesi Sosyal Bilimler Dergisi, 17(2), 21-36.

Guzel, A., \& Barin, E. (2013). Yabanci Dil Olarak Turkce Ogretimi. Ankara: Akcag Yayincilik.

Karababa, Z. C., Eker, D. N., \& Arik, R. S. (2010). Descriptive study of learner's level of autonomy: voices from the Turkish language classes. Procedia-Social and Behavioral Sciences, 9, 1692-1698.

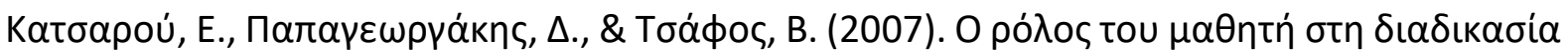

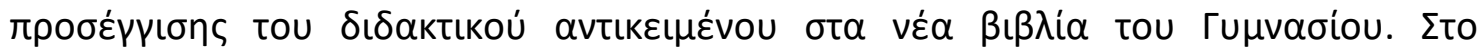

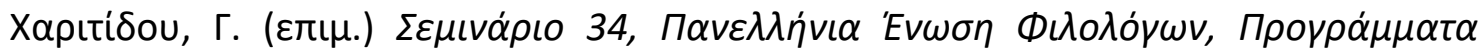

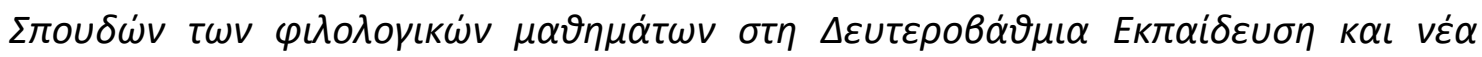
$\delta \iota \delta \alpha \kappa \tau \iota k \alpha \dot{~ B \iota B \lambda i \alpha . ~ A \theta \eta ́ v \alpha: ~ E \lambda \lambda \eta v o \varepsilon к \delta о \tau เ к n ́ . ~}$

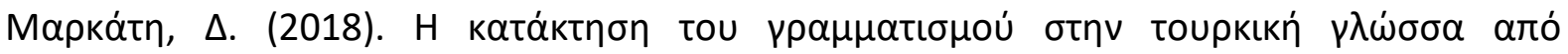

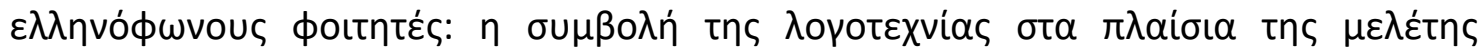

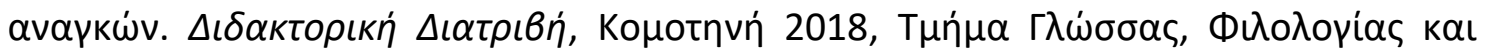

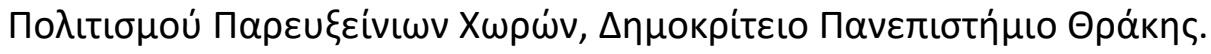

Memis, M., \& Erdem, M. D. (2013). Yabanci dil ogretiminde kullanilan yontemler, kullanim ozellikleri ve elestiriler. Turkish Studies, 8(9), 297-318.

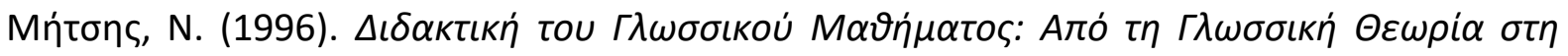
$\triangle \iota \delta \alpha \kappa \tau \iota \kappa \dot{~ П \rho \alpha ́ \xi \eta . ~ A \theta n ் v a: ~ G U T E N B E R G . ~}$

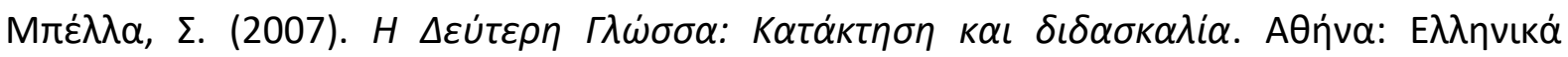
Гра́ $\mu \mu \alpha \tau \alpha$.

Prasad, N. (2013). Communicative Language Teaching in 21st Century ESL Classroom. English for Specific Purposes World, 40 (14), 1-8.

Rose, C., Wang, Y., Cui, Y., Arguello, J., Stegmann, K., Weinberger, A., \& Fischer, F. (2008). Analyzing collaborative learning processes automatically: Exploiting the advances of computational linguistics in computer-supported collaborative learning. International Journal of Computer-Supported Collaborative Learning, 3(3), 237-271. Available online at https://link.springer.com/article/10.1007/s11412-007-9034-0.

Sahin, Y. (2007). Yabanci Dil Ogretiminde Egitbilimsel ve Dilbilimsel Temelleri, Sosyal Bilimler Enstitusu Dergisi, 465-470.

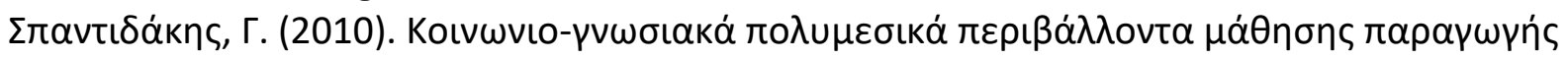

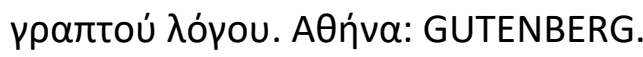


Tosun, F. P. (2008). Contextualized language instruction: Exploring the role of authentic animated stories on the attitudes of young EFL learners towards learning English (Unpublished MA Thesis). Cukurova University Institute of Social Sciences ELT Department, Adana, Turkey.

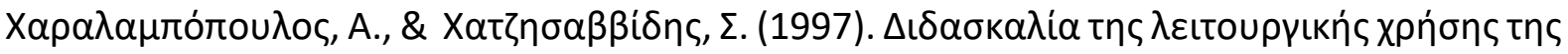

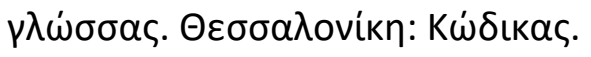

